\title{
Renalase, a catecholamine-metabolising enzyme?
}

\author{
F. Boomsma ${ }^{1}$, K. F. Tipton ${ }^{2}$ \\ ${ }^{1}$ Department of Internal Medicine, Section of Vascular Pharmacology, Erasmus MC, Rotterdam, The Netherlands \\ ${ }^{2}$ Department of Biochemistry, Trinity College, Dublin, Ireland
}

Received: September 6, 2006 / Accepted: October 12, 2006 / Published online: March 26, 2007

(C) Springer-Verlag 2007

\begin{abstract}
Summary Recently, a new FAD-dependent amine oxidase, renalase, was described. It was secreted by the kidney into the blood and shown to have significant cardiovascular actions, which were attributed to its catecholamine-metabolising activity. The authors concluded that renalase might be an important regulatory factor in human (patho)physiology. The catecholamine-metabolising activity of renalase in plasma contrasts with previous investigations where catecholamines were found to be stable in human plasma, provided autoxidation is prevented by an antioxidant. The claim of catecholamine-metabolising activity of renalase was based on the generation of $\mathrm{H}_{2} \mathrm{O}_{2}$ during incubation of the enzyme with catecholamines. Careful inspection and calculations of the data lead to the conclusion that the rate of $\mathrm{H}_{2} \mathrm{O}_{2}$ generation is far too low to be ascribed to enzymatic conversion of catecholamines by renalase. Renalase may well have important cardiovascular functions, but there is no proof that its actions are mediated through catecholamine-metabolising activity.
\end{abstract}

Keywords: Renalase, amine oxidase, catecholamines, metabolism, dopamine, noradrenaline

\section{Introduction}

Recently an intriguing paper described a new flavin-adenine dinucleotide (FAD) containing hormone, named renalase, which is secreted by the kidney and circulates in blood (Xu et al., 2005). Renalase was reported to be an amine oxidase with significant activity towards the catecholamines noradrenaline (NA), adrenaline (ADR) and dopamine (DA), but with little or no activity towards other physiologicallyoccurring amines, such as serotonin, tyramine, benzylamine, methylamine and spermidine. Although the amine oxidase activity of renalase was dependent on its FAD content, it was insensitive to the FAD-containing monoamine

Correspondence: Dr. F. Boomsma, Department of Internal Medicine, Section of Vascular Pharmacology, Erasmus MC, Dr. Molewaterplein 40, 3015 GD Rotterdam, The Netherlands

e-mail: f.boomsma@erasmusmc.nl oxidase (EC 1.4.3.4) inhibitors pargyline and clorgyline. Thus this reported amine oxidase activity of renalase differs in specificity and inhibitor-sensitivity from the known FAD-containing amine oxidases. Renalase was shown to have significant cardiovascular actions when injected into rats and these were ascribed to its catecholamine-metabolising activity. In patients with end-stage renal disease, renalase was found to be lacking from blood. The authors suggested that the explanation for the increased frequency of hypertension and cardiovascular dysfunction in these patients might be the higher plasma catecholamine concentrations resulting from the absence of renalase. They concluded that renalase might be an important regulatory factor in human (patho)physiology.

Since the presence of this novel catecholamine-metabolising enzyme in plasma is intriguing, but at variance with our own experience, we took a closer look at previously obtained data and at the evidence presented for the catecholamine-metabolising activity of renalase.

\section{Results and discussion}

Plasma of many species contains an enzyme, named semicarbazide-sensitive amine oxidase (SSAO; E.C.1.4.3.6), which catalyses the oxidative deamination of primary amines to form the corresponding aldehydes plus $\mathrm{H}_{2} \mathrm{O}_{2}$ and ammonia. The substrates converted depend on the species, but generally include methylamine and aminoacetone, which are believed to be the physiological substrates, as well as benzylamine and 2-phenylethylamine (Boomsma et al., 2003; Tipton et al., 2000). The SSAO in plasma of many species is also able to oxidize DA, to a much lesser extent, 
NA. ADR (a secondary amine) is not a substrate for SSAO in any of the species investigated. For example, after incubation of the catecholamines NA, DA and ADR in cow plasma for $4 \mathrm{~h}$ at $4{ }^{\circ} \mathrm{C} 95 \%$ of DA and $10 \%$ of NA had been metabolised. After $4 \mathrm{~h}$ at $20^{\circ} \mathrm{C}$ virtually all DA and $42 \%$ of NA had been metabolised. ADR was completely unchanged after incubation at either temperature. In the presence of semicarbazide, an inhibitor of SSAO, all conversions were completely blocked, with no loss of any of the catecholamines being observed (Boomsma et al., 1993). These results indicate that there is no catecholamine metabolising activity other than SSAO in plasma from this source.

The levels of SSAO in human plasma are relatively low and do not cause significant oxidation of any of the catecholamines. After incubation of NA, DA and ADR in human plasma for $4 \mathrm{~h}$ at either $4^{\circ} \mathrm{C}$ or $20^{\circ} \mathrm{C}$, all these catecholamines were found to be completely unaffected. The absence of oxidative activity towards DA was also confirmed by use of the radiochemical assay (Tipton et al., 2000) in platelet-free human plasma.

$\mathrm{Xu}$ et al. (2005) based their claim of the catecholamineoxidising activity of renalase on the generation of $\mathrm{H}_{2} \mathrm{O}_{2}$, detected by the Amplex Red Monoamine Oxidase Assay Kit (from Invitrogen), during incubation of the enzyme with NA, DA and ADR. The possibility that this activity might be due to plasma ceruloplasmin can be excluded, since the amine oxidase activity of that protein does not produce $\mathrm{H}_{2} \mathrm{O}_{2}$. These results are at variance with our observations, reported above. However, non-enzymatic autoxidation to which catecholamines are particularly prone at $\mathrm{pH}$ values above neutrality does result in the loss of catecholamines and the formation of $\mathrm{H}_{2} \mathrm{O}_{2}$. This autoxidation may be reduced by the addition of anti-oxidative agents, such as ascorbic acid or glutathione, but Xu et al. (2005) do not indicate that antioxidants were added in their experiments.

Inspection of the relevant figures in the paper by $\mathrm{Xu}$ et al. (2005) showed that 3.1, 2.5 and $1.8 \mathrm{nmol}_{2} \mathrm{O}_{2}$ were formed per mg per min from DA, ADR and NA, respectively, under the standard conditions of the assay that they used. Since their assay mixture contained $10 \mu \mathrm{g}$ of recombinant renalase, $2 \mathrm{mM}$ catecholamines, with a $30 \mathrm{~min}$ incubation at $20^{\circ} \mathrm{C}$, that would mean that $<1 \mathrm{nmol}$ of $\mathrm{H}_{2} \mathrm{O}_{2}$ is formed from $400 \mathrm{nmol}$ of catecholamine, which would correspond to a conversion of $0.25 \%$. Thus, the possibility that the observed $\mathrm{H}_{2} \mathrm{O}_{2}$ formation might arise from one or more contaminants common to the three catecholamines (which themselves have a purity of $<97-98 \%$ when commercially purchased) cannot be excluded.

Even if the formation of $\mathrm{H}_{2} \mathrm{O}_{2}$ were, indeed, caused by oxidation of catecholamines by renalase, this is clearly a very slow process. Unless the concentration of renalase in plasma would be very high (there is no mention in the paper of the actual concentrations) this could not have any physiological relevance, certainly in view of the fact that (patho)physiological plasma concentrations of catecholamines are at most a few $\mathrm{nM}$ for NA, and less than that for DA and ADR, i.e. a million-fold lower than the concentrations used in the experiments of $\mathrm{Xu}$ et al. (2005). Certainly, it is unlikely that effects seen in patients with end-stage renal disease result solely from the absence of such low catecholamine-oxidising activities or that the effects seen in the rat after injection of $0.5 \mathrm{mg}$ renalase (Xu et al., 2005) were simply a response to this activity.

We conclude that it is highly unlikely that renalase is a catecholamine-metabolising enzyme. The discovery of renalase is an impressive piece of work, and renalase may well have important cardiovascular functions, but these must be mediated through another mechanism. The search for the real substrate of this interesting flavo-enzyme should be continued.

\section{References}

Boomsma F, Alberts G, Bevers MM, Koning MM, Man in 't Veld AJ, Schalekamp MADH (1993) Breakdown of 3,4-dihydroxybenzylamine and dopamine in plasma of various animal species by semicarbazidesensitive amine oxidase. J Chromatogr 621: 82-88

Boomsma F, Bhaggoe UM, van der Houwen AMB, van den Meiracker AH (2003) Plasma semicarbazide-sensitive amine oxidase in human (patho)physiology. Biochim Biophys Acta 1647: 48-54

Tipton KF, Davey G, Motherway M (2000) Monoamine oxidase assays. In: Enna SJ, Willias M, Ferkany JW, et al. (eds) Current protocols in pharmacology. John Wiley New York, pp 3.6.1-3.6.42

Xu J, Li G, Wang P, Velazquez H, Yao X, Li Y, Wu Y, Peixoto A, Crowley S, Desir GV (2005) Renalase is a novel, soluble monoamine oxidase that regulates cardiac function and blood pressure. J Clin Invest 115: $1275-1280$ 